\title{
Caracterización de la textura de suelo en la subcuenca del río Zaratí para la evaluación del sistema de agua subterránea
}

\author{
Márquez, Karol \\ Facultad de Ingeniería Civil, Universidad Tecnológica de Panamá \\ Grupo de investigación Water Management and Hydrological Sciences, \\ Universidad Tecnológica de Panamá, Panamá \\ karol.marquez@utp.ac.pa \\ Carranza, Claudia \\ Facultad de Ingeniería Civil, Universidad Tecnológica de Panamá \\ Grupo de investigación Water Management and Hydrological Sciences, \\ Universidad Tecnológica de Panamá, Panamá \\ claudia.carranza@utp.ac.pa \\ Fábrega, José \\ Centro de Investigaciones Hidráulicas e Hidrotécnicas, Universidad Tecnológica de Panamá \\ Grupo de investigación Water Management and Hydrological Sciences, \\ Universidad Tecnológica de Panamá, Panamá \\ jose.fabrega@utp.ac.pa \\ Muñoz, Melisabel \\ Facultad de Ingeniería Civil, Universidad Tecnológica de Panamá \\ Grupo de investigación Water Management and Hydrological Sciences, \\ Universidad Tecnológica de Panamá, Panamá \\ melisabel.munoz@utp.ac.pa \\ Leiva, Jorge \\ Universidad de Costa Rica, Escuela de Agronomía, Sede de Guanacaste \\ Grupo de investigación Water Management and Hydrological Sciences, \\ Universidad Tecnológica de Panamá Cartago, Costa Rica \\ jleivatico@gmail.com
}

\section{ABSTRACT}

The soil texture is caracterized in the sub-basin of the Zaratí River (SRZ), in Cocle. The study area is in the Dry Arc region, where the effects of climate change are experienced, such as a longer dry season. Soil texture is a start to determine possible water recharge zones 
and characterize aquifers in the area. To determine the soil textures in the sub-basin, field trips were carried out to collect samples, which were subjected to sieving and hydrometer tests. The results were processed by two methods: a spreadsheet provided by the Materials Testing Laboratory of the Technological University of Panama and the Texture function in RStudio ${ }^{\circledR}$. Subsequently, the percentages of sand, silt and clay were plotted in the USDA texture triangle. Soil texture triangles divided by method of analysis and sub-basin zone were made. In the territory of the sub-basin, seven different soil textures were found, the most common in both methods being the sandy-clay-loam texture. Soil textures were compared for each sample processed in both methods and of 103 samples, 66 coincided in soil texture. The Texture function resulted being timesaving, as it required a fewer amount of input-data and multiple samples can be analyzed at the same time. On the other hand, several samples had to be discarded in the spreadsheet due to results that could not be used.

Keywords: RStudio, unsaturated hydraulic conductivity, Zaratí, hydrogeology, soil texture

\section{RESUMEN}

Se caracteriza la textura de suelo en la subcuenca del río Zaratí (SRZ), en Coclé. El área de estudio está localizada en el Arco Seco, donde se viven efectos del cambio climático, como temporada seca más prolongada. La textura de suelo es un inicio para determinar posibles zonas de recarga hídrica y caracterizar acuíferos en el área. Para determinar las texturas de suelo en la subcuenca se realizaron giras de campo para recolectar muestras a las que se le hicieron pruebas de tamizaje e hidrometría. Los resultados fueron procesados por dos métodos: una hoja de cálculo proporcionada por el Laboratorio de Ensayo de Materiales de la Universidad Tecnológica de Panamá y la función Texture en RStudio ${ }^{\circledR}$. Posteriormente, se graficaron los porcentajes de arena, limo y arcilla dentro del triángulo de textura del USDA. Se realizaron triángulos de textura de suelo divididos por método implementado y zona de la SRZ. En el territorio de la subcuenca se encontraron siete texturas de suelo diferentes, siendo la más común en ambos métodos la textura franco-arcillo-arenosa. Se compararon las texturas de suelo para cada muestra procesada en ambos métodos y de 103 muestras 66 coincidieron en textura de suelo. Con la función Texture se puede ahorrar tiempo por la menor cantidad de datos de entrada que requiere. Además, se pueden analizar múltiples pruebas a la vez. Por otra parte, en la hoja de cálculo se tuvieron que descartar varias muestras debido a resultados que no se pudieron utilizar.

Palabras claves: RStudio, conductividad hidráulica no saturada, Zaratí, Hidrogeología, textura de suelo. 


\section{INTRODUCCIÓN}

El estudio de aguas subterráneas está presentando más relevancia por la creciente demanda de agua dulce para el consumo humano y las actividades económicas. En Panamá, la región del Arco Seco presenta la reducción en la disponibilidad de aguas superficiales a causa de sequías que ocurrendurante la estación seca y que pueden ser prolongadas por efectos del cambio climático. Para poder estudiar los reservorios de aguas subterráneas se requiere el conocimiento de información acerca de los suelos del lugar, empezando con la textura de suelo. La subcuenca del río Zaratí presentadeficiencia en la distribución de agua por la falta de disponibilidad de agua en las fuentes superficiales, y esto, a su vez, afecta a las actividades económicas de la zona como lo es la agricultura. Es por esto que esta investigación presenta los procesos implementados para la generación de las texturas de suelo de la SRZ como información necesaria para los estudios de las posibles zonas de recarga de acuíferos de esta región.

\section{METOdOLOGÍA}

\section{A. Trabajo en campo}

Se realizaron giras en las estaciones seca y lluviosa donde se visitaron 18 sitios seleccionados en lasubcuenca del río Zaratí en las zonas baja, media y alta. Se buscó que la cantidad de sitios por zona fuese proporcional a la superficie de la zona. En los lugares de muestreo se recolectaron muestras de suelo de aproximadamente $1 \mathrm{~kg}$.

\section{B. Pruebas de laboratorio}

Para identificar la textura de suelo se realizaron dos pruebas distintas: tamizaje e hidrometría. La prueba de tamizaje se realizó siguiendo los lineamientos de la norma ASTM-D6913 [1] donde se separaron las partículas de suelo lavado y secado en horno de acuerdo a sus diámetros y se pesó el suelo retenido en cada tamiz. Para la prueba de hidrometría se tomó como referencia la norma ASTM- D7928 [2]. La prueba distingue los tamaños de partículas finas presentes en una muestra de 50 gramos de suelo previamente pasado por el tamiz $\mathrm{N}^{\circ}$ 10 y reposado en solución de hexametafosfato al4\% utilizada para evitar la floculación de partículas. Las mediciones se realizaron con el hidrómetro de modelo $152 \mathrm{H}$ en diferentes intervalos desde 1 minuto hasta 24 horas. Se hizo este análisis para 103 muestras de suelo.

\section{Procesamiento de datos}

Los resultados de las pruebas realizadas en laboratorio se procesaron utilizando dos métodos: uno fue la hoja de cálculo en Excel proporcionada por el Laboratorio de Ensayo de Materiales [3] y el otro fue la función Texture del paquete Envalysis de la herramienta RStudio ${ }^{\circledR}[4]$. En la hoja de cálculo se introdujeron los resultados de las pruebas de tamizaje 
e hidrometría y se obtuvo como resultado una gráfica semilogarítmica de porcentaje de suelo que pasa vs tamaño de partícula (escala logarítmica). Apartir de la gráfica se tienen los porcentajes de grava, arena, limo y arcilla. Se analizó una muestra por cada hoja de cálculo.

La función Texture solamente requirió de los resultados de la prueba de hidrometría y permitía analizar múltiples muestras a la vez. Con los datos mencionados hizo una estimación de los porcentajes de arena, limo y arcilla al ajustar los puntos obtenidos (porcentaje que pasa, tamaño de partícula) a una de varias opciones de curvas estadísticas existentes.

Los porcentajes de arena, limo y arcilla son utilizados para determinar la textura de suelo. Se escogió clasificar según los parámetros del Departamento de Agricultura de Estados Unidos (USDA). Con el paquete SoilTexture en RStudio ${ }^{\circledR}$ se grafican los porcentajes dentro del triángulo de textura delUSDA.

\section{RESULTADOS}

\section{A. Triángulos de textura}

Se crearon seis triángulos de textura (Fig.1 - 3) para la SRZ, tomando en cuenta las partes de la subcuenca y los dos métodos de procesamiento de datos. En ellos se encontró que de 12 texturas de suelo en la clasificación del USDA, la subcuenca del río Zaratí presenta siete clases texturales. Estas clases son: franco arcillo arenosa, franco arenosa, franco arcillosa, arcilla, arcillo arenosa, franco y arena franca. En el método donde se utilizó la hoja de cálculo del LEM hubo que descartar algunas pruebas por tener resultados que no se pudieron graficar en los triángulos de textura (Fig.1 - 3).

a)

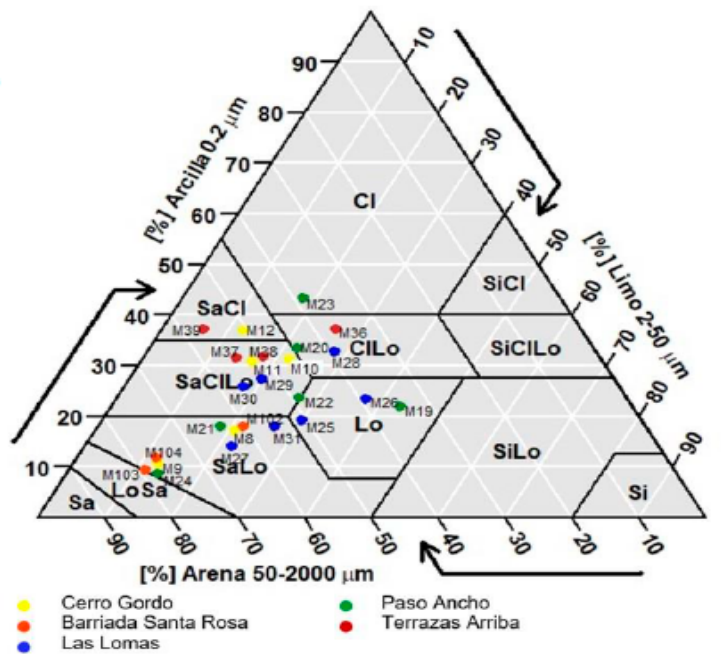

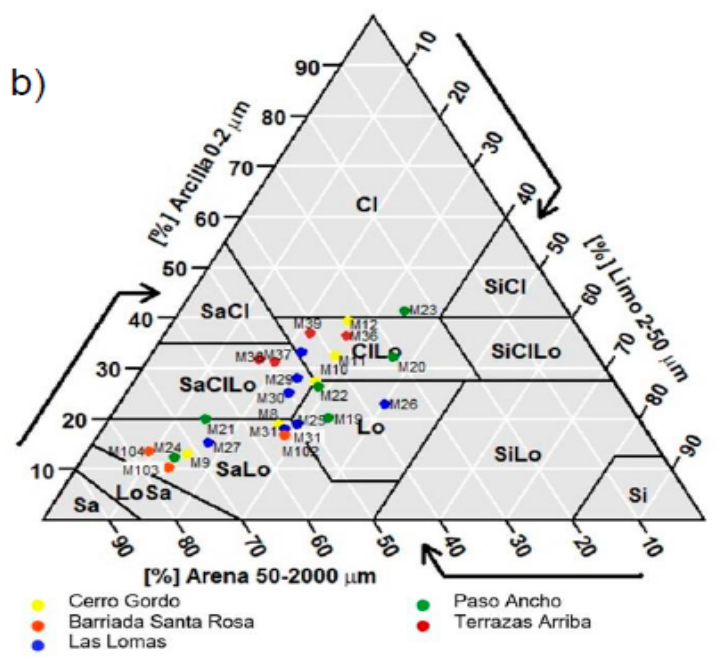

b)

Fig. 1: Triángulos de textura de suelo de la zona baja de a) datos normalizados y b) función Texture, respectivamente. 
a)

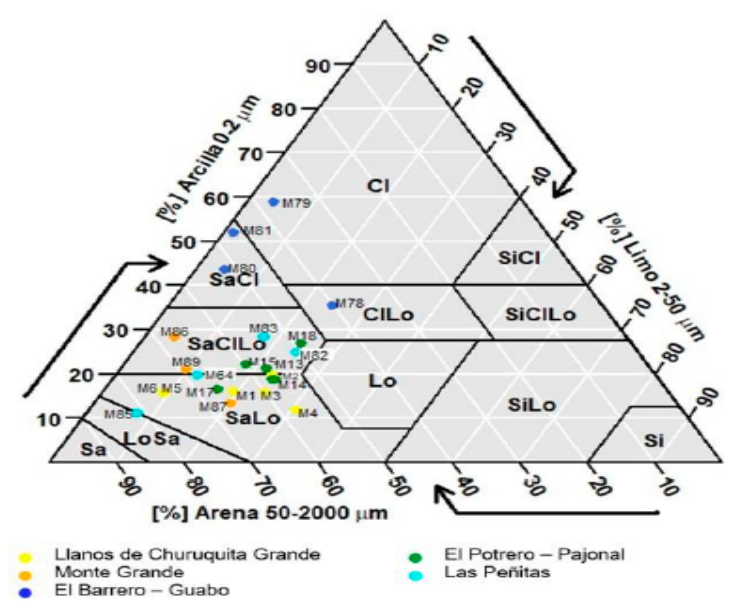

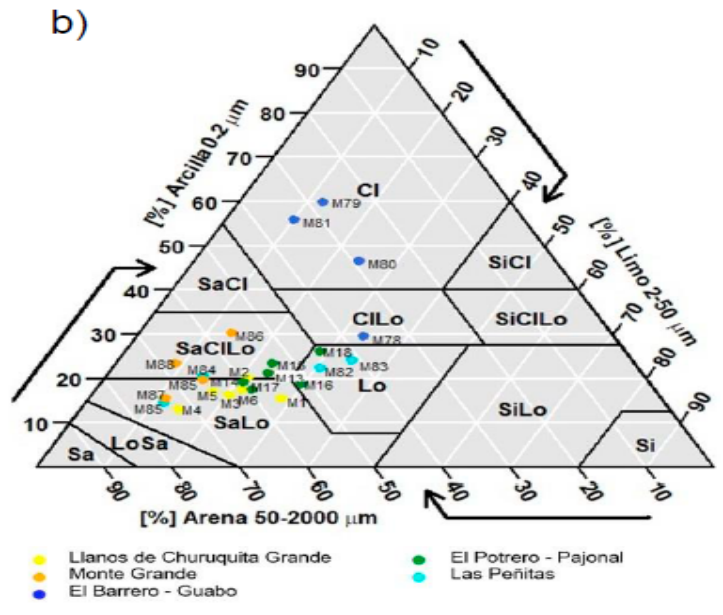

Fig. 2: Triángulos de textura de suelo de la zona media de a) datos normalizados y b) función Texture, respectivamente.

a)

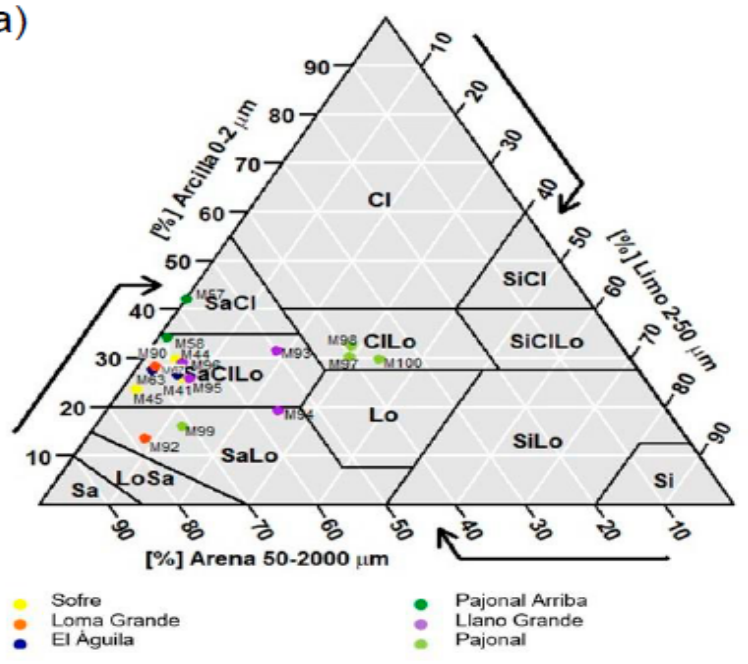

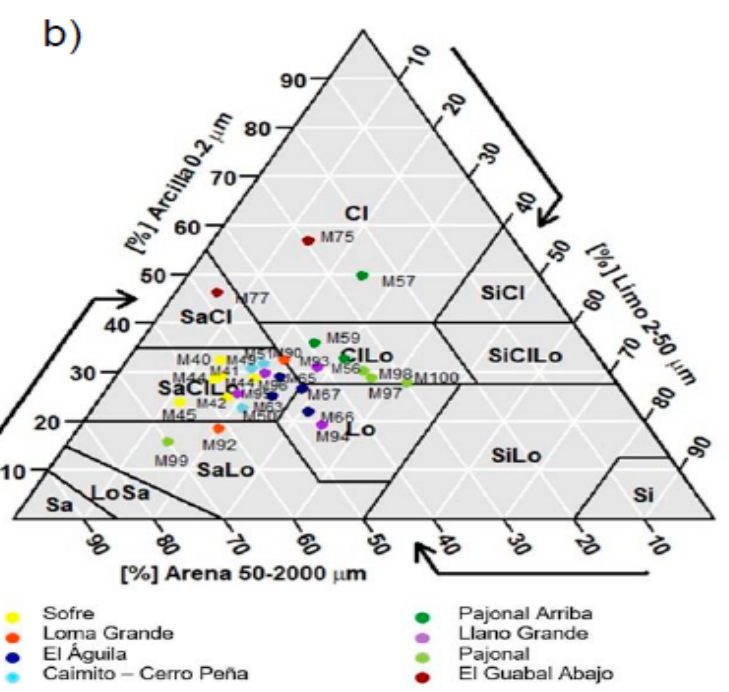

Fig. 3: Triángulos de textura de suelo de la zona alta de a) datos normalizados y b) función Texture, respectivamente. 
Tabla 1. Texturas de suelo de la SRZ y su distribución en los métodos implementados

\begin{tabular}{ccc}
\hline Textura de suelo & Datos normalizados & Función Texture \\
\hline Franco arcillo arenoso & $25,24 \%$ & $22,33 \%$ \\
Franco arenoso & $23,30 \%$ & $22,33 \%$ \\
Franco arcilloso & $6,80 \%$ & $14,56 \%$ \\
Arcilla & $1,94 \%$ & $5,83 \%$ \\
Arcillo arenoso & $4,85 \%$ & $0,97 \%$ \\
Franco & $2,91 \%$ & $9,71 \%$ \\
Arena franca & $0,97 \%$ & $0 \%$ \\
\hline
\end{tabular}

Para los datos realizados con la hoja de cálculo, se considera probable que la cantidad de muestrasque no se pudieron utilizar haya afectado los valores de porcentajes de textura de suelo en la subcuenca. La textura que se presentó con mayor frecuencia fue franco arcillo arenosa. La textura franco-arenosa se presentó con mayor incidencia en la zonas baja y media, y la textura franco arcillo arenosa presentó mayor incidencia en la zona alta de la SRZ.

\section{CONCLUSIONES}

Los dos métodos para determinar los porcentajes de arena, limo y arcilla presentaron diferentes grados de efectividad. Por una parte, la hoja de cálculo solo funciona para una muestra a la vez mientras que en RStudio ${ }^{\circledR}$ se pueden analizar todas las muestras a la vez. Adicionalmente, con el segundo método no hubo que descartar ninguna muestra como ocurrió en las hojas de cálculo. Si bien la función Texture de RStudio es muy reciente, se compararon los dos grupos de datos; de 103 muestras analizadas, 66 de ellas coincidieron en texturas de suelo parecidas en los dos métodos. Enla SRZ se encontraron texturas de suelo con contenidos considerables de arena, la cual se caracteriza por permitir con mayor facilidad del flujo de agua en el suelo. Con estos resultados de las texturas de las capas superficiales del suelo de la SRZ, se puede iniciar el reconocimiento de posibles zonas de recarga hídrica en la subcuenca.

\section{Referencias}

[1] ASTM International, "Standard Test Methods for Particle-Size Distribution (Gradation ) of Soils Using Sieve Analysis D6913/D6913M," ASTM Int. West Conshohocken, PA, vol. 04, no. Reapproved 2009, pp. 1-35, 2004, doi: 10.1520/D6913-04R09.2.

[2] ASTM International, "Standard Test Method for Particle-Size Distribution (Gradation) of Fine- Grained Soils Using the Sedimentation (Hydrometer) Analysis D7928," ASTM Int. West Conshohocken, PA., no. May 2016, pp. 1-25, 2017, doi: 10.1520/D7928-17.

[3] LEM, “Hoja de cálculo de análisis granulométrico." Panamá, 2019.

[4] "RStudio | Open source \& professional software for data science teams - RStudio." [Online]. Available: https://rstudio.com/. [Accessed: 17-May-2020]. 


\section{Autorización y Licencia CC}

Los autores autorizan a APANAC XVIII a publicar el artículo en las actas de la conferencia en Acceso Abierto (Open Access) en diversos formatos digitales (PDF, HTML, EPUB) e integrarlos en diversas plataformas online como repositorios y bases de datos bajo la licencia CC:

Attribution-NonCommercial-ShareAlike 4.0 International (CC BY-NC-SA 4.0) https://creativecommons. org/licenses/by-nc-sa/4.0/.

Ni APANAC XVIII ni los editores son responsables ni del contenido ni de las implicaciones de lo expresado en el artículo. 